\title{
Topografia do cone medular do cachorro-do-mato (Cerdocyon thous Linnaeus, 1766): relato de caso
}

\author{
Luane Lopes Pinheiro ${ }^{1 *}$ \\ Ana Rita de Lima ${ }^{1}$ \\ Ana Carla Barros de Souza ${ }^{2}$ \\ Luiza Corrêa Pereira ${ }^{2}$ \\ Érika Branco ${ }^{1}$ \\ ${ }^{1}$ Universidade Federal Rural da Amazônia - UFRA. \\ Avenida Presidente Tancredo Neves, 2501, \\ CEP 66.077-530, Belém - PA, Brasil \\ ${ }^{2}$ Mina de Bauxita - Empresa Terra LTDA. Paragomina - PA, Brasil \\ *Autor para correspondência \\ luanelopespinheiro@gmail.com
}

Submetido em 04/04/2011

Aceito para publicação em 12/09/2011

\section{Resumo}

O cachorro-do-mato é o canídeo mais comum do continente sul-americano. De modo geral, tem sua dieta variada conforme a época do ano e a região habitada, o que permite a este uma ampla distribuição geográfica. Junto ao interesse na anatomia comparada, o presente trabalho objetivou descrever a topografia do cone medular do cachorro-do-mato com intuito de oferecer informações que possam dar subsídios para a prática de anestesia epidural, cuja eficiência depende do conhecimento da anatomia desta região. Foi utilizado um espécime, macho, adulto, proveniente da Mina de Bauxita - Paragominas-PA, o qual foi dissecado na região lombo-sacral. O cone medular do cachorro-do-mato apresentou base ao nível de L6 e ápice em S3, apresentando comprimento de $10,13 \mathrm{~cm}$. Sugerimos que a região sacrococcígea é a mais indicada para a anestesia epidural, considerando que o exemplar em estudo apresentou nove vértebras lombares e quatro vértebras sacrais.

Palavras-chave: Anatomia, Anestesia epidural, Medula espinhal

\section{Abstract}

Topography of the medullary cone of the crab-eating fox (Cerdocyon thous Linnaeus, 1766): Case report. The crab-eating fox is the most common Canidae of South America. In general, its diet varies according to the season and inhabited region. In this study, the medullary cone of the crab-eating fox was described because of interests in comparative anatomy, with the goal of providing information that could assist in epidural anesthesia, which cannot be efficiently practiced without knowledge of this anatomical region. We investigated an adult male from the Bauxite Mine (Paragominas, PA), which was dissected in the lumbosacral region. The medullary cone was $10.13 \mathrm{~cm}$ long; the base began at the L6 and the apex was at the S3. Considering that the specimen studied had nine lumbar and four sacral vertebrae, we conclude that the sacrococcygeal region is probably the most suitable place for epidural anesthesia.

Key words: Anatomy, Epidural anesthesia, Spinal cord 


\section{Introdução}

O cachorro-do-mato (Cerdocyon thous Linnaeus, 1766), também conhecido como guaraxaim, pertence à Ordem Carnívora e Família Canidae, sendo uma espécie amplamente distribuída pela América do Sul, que com hábitos noturnos e crepusculares, vive solitário ou em pares, explorando áreas de mata e/ou savana (EMMONS, 1999).

Tais animais são de médio porte, quando adultos pesam de 6 a $7 \mathrm{~kg}$ medindo cerca de $65 \mathrm{~cm}$ de comprimento. Apresentam pelo relativamente curto e pelagem cinza com alguns pelos negros, sendo alguns indivíduos de coloração mais amarelada e outros quase negros. Alimentam-se de insetos e pequenos vertebrados e invertebrados, além de frutos, tendo preferência por pequenos roedores (RAMOS JR. et al., 2003).

O cachorro-do-mato, salvo aspectos gerais, tem sua morfologia pouco conhecida na literatura havendo necessidade de ampliação dessas informações, especialmente as que são de interesse veterinário ou que possam contribuir para preservação da espécie.

A medula espinhal e suas raízes nervosas são o local de ação de numerosos fármacos. A anestesia epidural, também conhecida por anestesia peridural ou extradural é obtida através da injeção de anestésicos no espaço epidural o qual é compreendido entre o periósteo do canal vertebral e a dura-máter (INTELIZANO et al., 2002; OTERO, 2005).

A anestesia epidural é uma técnica anestésica consagrada, que tem como principais vantagens a segurança, eficiência e baixo custo (HALL, 1992; MUIR et al., 2001). Tem sido utilizada com frequência na clínica de cães e gatos com a finalidade de uma ação regionalizada de grande eficácia, que permite a anestesia e/ou analgesia de uma determinada região para que sejam possíveis procedimentos cirúrgicos, diagnósticos e recuperações pós cirúrgicas e pós traumáticas com analgesia satisfatória. Além disso a ausência quase total de depressão cárdio-respiratória favorece sua indicação como técnica anestésica alternativa para animais de alto risco, no qual a anestesia geral estaria contra-indicada (BEDNARSKI, 1996; OTERO, 2005).
A terminação da medula espinhal varia entre as espécies, e a correta realização da anestesia peridural depende do conhecimento anatômico das estruturas que compõem o canal vertebral. $\mathrm{O}$ acesso epidural, por exemplo, tanto em caninos quanto em felinos, geralmente é realizado no espaço lombossacro (L7-S1), haja visto que o espaço epidural é mais volumoso de acordo com o afilamento e término da medula espinhal (cone medular) (INTELIZANO et al., 2002; OTERO, 2005).

Tendo em vista que o local de aplicação de medicamentos anestésicos varia conforme a espécie animal, de acordo com o local do término da medula espinhal (DYCE et al., 2004; FANTONI; CORTOPASSI, 2002), o presente estudo teve como objetivo descrever a topografia do cone medular do cachorro-do-mato (Cerdocyon thous Linnaeus, 1766), uma vez que esse conhecimento assume considerável importância no contexto geral da anestesia e aplicação de diferentes fármacos.

\section{Material e Métodos}

Para o presente estudo foi utilizado um espécime criopreservado de cachorro-do-mato (Cerdocyon thous Linnaeus, 1766), macho, adulto, que foi a óbito por causas naturais, sendo este proveniente de ParagominasPA, sob autorização SEMA-PA No 455/2009 e 522/2009, doado ao Instituto de Saúde e Produção Animal ISPA, da Universidade Federal Rural da Amazônia - UFRA. A dissecação foi realizada imediatamente após o descongelamento do animal, mediante incisão e afastamento da pele na linha mediana dorsal, desde a região torácica cranial até a base da cauda, prosseguindo com remoção da musculatura epiaxial e retirada dos arcos vertebrais com auxílio de costótomo, assim expondo a medula espinhal e seus envoltórios.

Com a identificação das vértebras regionais, a intumescência lombar e o cone medular foram individualizados enfatizando seu início (base) e seu término (ápice) em relação às vértebras lombares $\mathrm{e}$ sacrais e mensurados com auxilio de paquímetro digital Dc-6 Western.

Toda nomenclatura adotada foi baseada na International Committee on Veterinary Gross Anatomical Nomenclature (2005). 


\section{Resultados e Discussão}

O cachorro-do-mato (Cerdocyon thous Linnaeus, 1766) apresentou nove vértebras lombares e quatro vértebras sacrais, sendo que a base do cone medular localizou-se ao nível da $6^{\text {a }}$ vértebra lombar (L6) e o ápice ao nível da $3^{\underline{a}}$ vértebra sacral (S3), apresentando um comprimento de 10,13cm (Figura 1) (Crown-rump de $51 \mathrm{~cm})$.

Para o cão doméstico, Evans e De Lahunta (2001) descreveram que o cone medular tem sua base formada pelo sétimo segmento lombar da medula espinhal (L7) e que seu ápice é constituído pelo último segmento caudal (Ca5). Ainda em relação ao cão, segundo Santiago (1974) a base do cone medular se encontra na altura de L3 e seu ápice é registrado em L7. Fletcher (1979), no entanto, afirma que em cães de pequeno porte a extremidade do cone medular encontra-se na altura de L6, enquanto que em cães de grande porte acha-se junto à margem cranial de L7. Dessa forma, o cachorro-domato não se assemelha ao cão doméstico na topografia do cone medular.

Em comparação ao cachorro-do-mato-deorelhas-curtas (Atelocynus microtis), espécie que geograficamente encontra-se distribuida pela bacia amazônica e facilmente diferenciada dos outros canídeos neotropicais pelo tamanho da orelha, comprimento do corpo e coloração, Mançanares et al. (2010) relatam em um espécime jovem, com Crown-rump de $38 \mathrm{~cm}$, o cone medular medindo cerca de $3,9 \mathrm{~cm}$, com base em L7 e ápice em S3, assemelhando-se ao cachorro-do-mato de nosso estudo (base em nível de L6 e ápice em S3), porém com menor extensão em relação ao comprimento do cone medular $(10,13 \mathrm{~cm})$, considerando o tamanho dos animais.

Ainda em relação aos carnívoros não-domésticos, a topografia do cone medular do cachorro-do-mato assemelha-se mais com a descrita para o gato-mourisco (base em nível de L6 e ápice em S2) (CARVALHO et al., 2003), quati (base em nível de L5-6 e ápice em S3) (GREGORES et al., 2010) e Jaguatirica (base em nível de L4 e ápice em S3) (BRÍGIDA et al., 2010), em comparação com a descrita para o lobo-guará (base em nível de L3 e ápice em L5-6) (MACHADO et al., 2002) e ariranha (base em nível de L2 e ápice em L4) (MACHADO et al., 2009a). O lobo-marinho, por sua vez, segundo Machado et al. (2003) apresenta uma localização inusitada do cone medular entre as vértebras T5 e T7, caracterizando uma topografia totalmente diferente.

Quando comparado com registros de outros animais silvestres, os limites do cone medular do cachorro-domato diferem do tamanduá-mirim (base em nível de L1 e ápice em L5-6) (MACHADO; SANTOS, 2008), do sagui Callithrix jacchus jacchus (base em nível de L2 e ápice em L4) (MACHADO et al., 1992), da preguiça Choloepus hoffmanni (base em nível de L3 e ápice em L4) e a Bradypus variegatus (base em nível de L4 e ápice

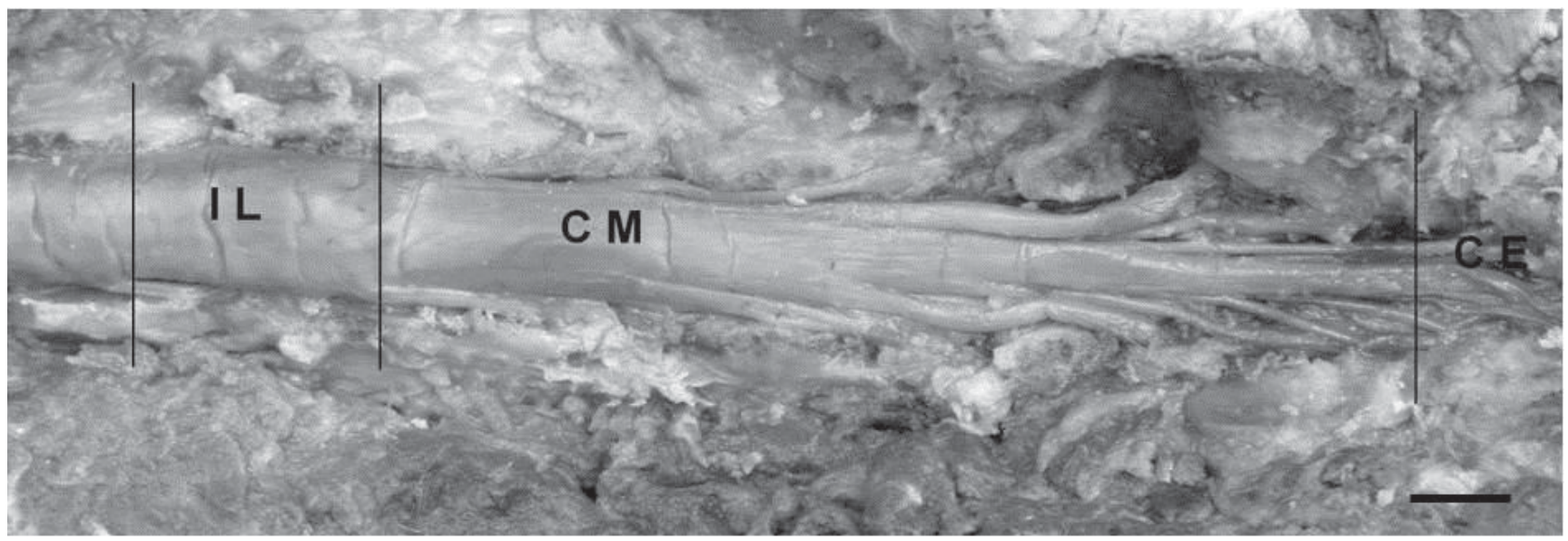

FIGURA 1: Fotomacrografia da vista dorsal do cone medular do cachorro-do-mato (Cerdocyon thous Linnaeus, 1766). IL - intumescência lombar; $\mathrm{CM}$ - cone medular; $\mathrm{CE}$ - cauda equina. Barra de escala: $1 \mathrm{~cm}$. 
em S1) (SILVA et al., 2002), além dos roedores silvestres, como a paca (base em nível de L5-7 e ápice em S1) (SCAVONE et al. 2007) e o ratão-do-banhado (base em nível de L3-5 e ápice em L5) (MACHADO et al., 2009b) que apresentam um cone medular relativamente curto.

No que se refere ao valor obtido do comprimento do cone medular do cachorro-do-mato, este se apresentou bem extenso $(10,13 \mathrm{~cm})$ quando comparado aos valores encontrados em jaguatirica, 8,5cm (BRÍGIDA et al., 2010); tamanduá mirim, 6,5 a 7,0cm (MACHADO; SANTOS, 2008); lobo guará, 6,5cm (MACHADO et al., 2002); quati, 5,2 a 5,8cm (GREGORES et al., 2010); ariranha, 5,5cm (MACHADO et al., 2009a); gato mourisco, 5cm (CARVALHO et al., 2003); lobomarinho, 4,40cm (MACHADO et al., 2003); paca, $3,39 \mathrm{~cm}$ (SCAVONE et al., 2007); e ratão do banhado, 2cm (MACHADO et al., 2009b).

A partir dos resultados foi possível concluir que o cone medular do cachorro-do-mato dissecado (Cerdocyon thous Linnaeus, 1766) localizou-se entre a $6^{\mathrm{a}}$ vértebra lombar e a $3^{\mathrm{a}}$ vértebra sacral, com comprimento de $10,13 \mathrm{~cm}$. Pela maior extensão do cone medular, sugere-se, portanto, que a anestesia epidural, nesses animais, seja realizada na região sacrococcígea, considerando que o uso desta técnica permite cirurgias e outros procedimentos não cirúrgicos com analgesia satisfatória e efeitos colaterais amplamente diminuídos.

\section{Agradecimentos}

Agradecemos à Mina de Bauxita, Paragominas-PA pela doação do animal ao Instituto de Saúde e Produção Animal - ISPA, da Universidade Federal Rural da Amazônia - UFRA.

\section{Referências}

BEDNARSKI, R. M. Anesthesia and immobilization of specific species - dogs and cats. In: JONES, L. (Ed.). Veterinary Anesthesia. 3. ed. Baltimore: Williams \& Wilkins, 1996. p. 591598.

BRÍGIDA, S. S. S.; BRANCO, E.; PINHEIRO, L. L.; MARTINS, D. M.; ARAÚJO, E. B.; MELUL, R.; LACRETA JR., A. C. C.; MENESES, A. M. C.; SOUZA, A. C. B.; PEREIRA, L. C.; FIORETTO, E. T.; LIMA, A. R. Topografia do cone medular da jaguatirica (Leopardus pardalis). Acta Veterinária Brasilica, Mossoró, v. 4, n. 1, p. 51-54, 2010.

CARVALHO, S. F. M.; SANTOS, A. L. Q.; AVILA JR., R. H.; ANDRADE, M. B.; MAGALHÃES, L. M.; MORAES, F. M.; RIBEIRO, P. I. R. Topografia do cone medular em um gato-mourisco, Herpailurus yagouaroundi (Severtow, 1858) (FELIDAE). Archives of Veterinary Science, Curitiba, v. 8, n. 2, p. 35-38, 2003.

DYCE, K. M.; SACK, W. O.; WENSING, C. J. G. Tratado de anatomia veterinária. 3. ed. Rio de Janeiro: Guanabara Koogan, 2004. $813 \mathrm{p}$.

EMMONS, L. H. Neotropical rainforest mammals: a field guide. Chicago: The University of Chicago Press, 1999. 306 p.

EVANS, H. E.; DELAHUNTA, A. Guia para a dissecação do cão. 5. ed. Rio de Janeiro: Guanabara Koogan, 2001. 250 p.

FANTONI, D. T.; CORTOPASSI, S. R. G. Anestesia em cães e gatos. 2. ed. São Paulo: Roca, 2002. 389 p.

FLETCHER, T. F. Spinal cord and meninges. In: EVANS, H. E.; CHRISTENSEN, G. C. (Ed.). Anatomy of the dog. 2 ed. Philadelphia: W.B. Saunders Company, 1979. 947 p.

GREGORES, G. B.; BRANCO, E.; CARVALHO, A. F.; SARMENTO, A. A. P.; OLIVEIRA, P. C.; FERREIRA, G. J.; CABRAL, R.; FIORETTO, E. T.; MIGLINO, M. A; CORTOPASSI, S. R. G. Topografia do cone medular do quati (Nasua nasua Linnaeus, 1766). Biotemas, Florianópolis, v. 23, n. 2, p. 173-176, 2010.

HALL, L. W. Princípios generales de la anestesia. In: HILBERY, A. D. R. (Ed.). Manual de anestesia de los pequeños animales. 3. ed. Zaragoza: Acribia, 1992. p. 1-8.

INTELIZANO, T. R.; SANTOS, P. R.; FUTEMA, F.; OTSUKI, D. A.; ALMEIDA, T. I. Técnicas de Anestesia Local. In: FANTONI, D. T.; CORTOPASSI, S. R. G. (Ed.). Anestesia em cães e gatos. 1. ed. São Paulo: Roca, 2002. p. 199-208.

INTERNATIONAL COMMITTEE ON VETERINARY GROSS ANATOMICAL NOMENCLATURE. Nomina Anatomica Veterinária. 5. ed. Hannover: Editorial Committee, 2005. 165 p.

MACHADO, G. V.; CAL, J. A.; BIRCK A. J. Topografia do cone medular no ratão-do-banhado (Myocastor coypus Molina, 1782 Rodentia: Mammalia). Biotemas, Florianópolis, v. 22, n. 2, p. 117120, 2009b.

MACHADO, G. V.; LESNAU, G. G.; BIRCK, A. J. Topografia do cone medular no lobo-marinho (Arctocephalus australis Zimmermann, 1803). Arquivos de Ciências Veterinárias e Zoologia da UNIPAR, Umuarama, v. 6, n.1, p.11-14, 2003.

MACHADO, G. V.; RIELlA, A. C. M.; LIEGEL, S. R. Esqueletopia do cone medular em Callithrix jacchus jacchus. In: CONGRESSO BRASILEIRO DE MEDICINA VETERINÁRIA, 22, 1992, Curitiba. Resumos... Curitiba: SBMV, 1992. p. 56.

MACHADO, G. V.; ROSAS, F. C. W.; LAZZARINI, S. M. Topografia do cone medular na ariranha (Pteronura brasiliensis Zimmermann, 1780). Ciência Animal Brasileira, Goiânia, v. 10, n. 1, p. 301-305, 2009a.

MACHADO G. V.; SANTOS B. S. Topografia do cone medular no tamanduá-mirim (Tamandua tetradactyla Linnaeus, 1758) (Xenarthra: Myrmecophagidae). Archives of Veterinary Science, Curitiba, v. 13, n. 3, p. 172-175, 2008.

MACHADO, V. M.; FONSECA, C. C.; DAS NEVES, M. T. D.; DE PAULA, T. A. R.; BENJAMIN, L. A. Topografia do cone medular 
no lobo-guará (Chrysocyon brachyurus Illiger, 1815). Revista Brasileira de Ciência Veterinária, Niterói, v. 9, n. 2, p.107-109, 2002.

MANÇANARES, A. C. F.; SADANHA, K. L.; BRANCO, E.; SOUZA, A. C. B.; PEREIRA, L. C.; LIMA, A. R. Topografia do cone medular do cachorro-do-mato-de-orelhas-curtas (Atelocynus microtis). In: CONGRESSO BRASILEIRO DE ANATOMIA, XXIV, 2010, Ribeirão Preto. Resumos... Ribeirão Preto: CBA, 2010, p. 371.

MUIR, W. W.; HUBBELL, J. A.; SKARDA, R. T.; BEDNARSKI, R. M. Manual de Anestesia Veterinária. 3. ed. Porto Alegre: Artes Médicas, 2001. 432 p.

OTERO, P. Administração epidural e espinhal de analgésicos. In: OTERO, P. (Ed.). Dor avaliação e tratamento em pequenos animais. São Caetano do Sul: Interbook, 2005. p. 192-210.

RAMOS JR., V. A.; PESSUTTI, C.; CHIEREGATTO, C. A. F. S. Guia de identificação dos canídeos silvestre brasileiro. v. 4 . Sorocaba: JoyJoy Studio Ltda, 2003. 35 p.
SANTIAGO, W. Esqueletopia do cone medular em Canis familiaris. Arquivo da Universidade Federal Rural do Rio de Janeiro, Seropédica, v. 4, n. 1, p. 67-69, 1974.

SCAVONE, A. R. F.; GUIMARÃES, G. C.; RODRIGUES, V. H. V.; SASAHARA, T. H. C.; MACHADO, M. R. F. Topografia do cone medular da paca (Agouti paca, Linnaeus - 1766). Brazilian Journal of Veterinary Research and Animal Science, São Paulo, v. 44, p. 53-57, 2007.

SILVA, D. R.; AMORIM JUNIOR, A. A.; AMORIM, M. J. A. A. L.; ANDRADE, M. B.; ARAUJO, F. P.; PIMENTEL, D. S. Topografia do cone medular da preguiça, Choloeppus hoffmanni PETERS, 1865 e Bradypus variegatus SCHINZ, 1825. In: CONGRESSO DE INICIAÇÃO CIENTÍFICA, 2, 2002, Recife. Resumos... Recife: Pro-Reitoria de Pesquisa e de Pós-Graduação da Universidade Federal Rural de Pernambuco, 2002. p. 527-528. 